\title{
Synthesis of Organo-soluble Tetrapyrazinoporphyrazines and Their Visible Spectra Properties
}

\author{
Chun Keun Jang, Song Hak Kim, Do Kyung Lee, and Jae-Yun Jaung

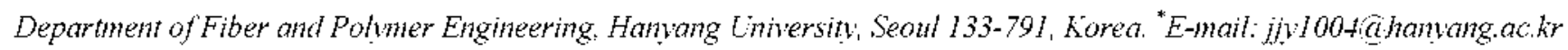 \\ Received Jume 30,2008
}

Key Words : 2.3-Diç̧anopy razine. Tetrapyrazinoporphỵrazine. tert-Butyl group. Fluorescence. Spectral properties

There were many researches about phthalocyanines for various applications such as chenical sensor. ${ }^{1}$ cataly st, ${ }^{2}$ etc. The unsubstituted metallophthalocyanines have intense colors but are generally insoluble in organic solvents or water, thereby limiting their use to just a few fields. It is well known that the insolubility of metal phthalocyanine derivatives results from their molecular stacking. which gives rise to strong intermolecular interactions between the macrocycles in the phthalocyanine molecules. There are. however. two methods of achieving solubility in almost all solvents. The first involves the use of a tri- or tetravalent transition metal as the core of the macrocycle. with the coordination compound having an axial ligand at the central metal. ${ }^{3}$ The second method involves the introduction of various peripheral substituents on the macrocycle. ${ }^{4}$ This minimizes the formation of aggregates. thereby improving the solubility.

We have been studied the syntheses of functional dye materials based on 2.3-dicyanopyrazine chromophores, and correlated their physical properties with structures. ${ }^{6}$ In this paper. we designed and synthesized metal and metal-free tetrapyrazinoporphyrazines derived from 2.3-dicyano-5-(4- tert-butylphenyl)-6-alkỵl pyrazines derivatives for increasing solubility.

Treatment of these 4-(tent-butyl)alkyl phenone derivatives ${ }^{7}$ with one equivalent of bromine in chloroform at room temperature gave $\alpha$-brominated 4-(tert-butyl)alkyl phenone derivatives. $\alpha$-Acetoxylated 4-(tert-butyl)alkyl phenone (obtained by the treatment of the $\alpha$-brominated ketone with an excess of anlyydrous potassium acetate in acetone) was reacted with $10 \%$ methanolic $\mathrm{NaOH}$ under reflux conditions to produce the corresponding compounds (2). The $\alpha$-diketones (3) were obtained by the oxidation of $\alpha$-hydrosyketone with copper sulfate in aqueous pyridine solution. Tetrapyrazinoporphyrazine precursors (4) were synthesized from $a$-diketones and diaminomaleonitrile. in the presence of a catalytic amount of $p$-toluenesulfonic acid in methanol.

The final tetrapyrazinoporphyrazine magnesium complexes (5) were successfully synthesized using excess magnesium butoxide in $n$-butanol under reflux conditions. ${ }^{5}$ These magnesiun complexes were easily demetalated. This was done by stirring them in excess $p$-toluenesulfonic acid. in THF, at room temperature, for $30 \mathrm{~min}$. to produce 6 (in a yield range of $44-50 \%$ ). The reaction route is summarized

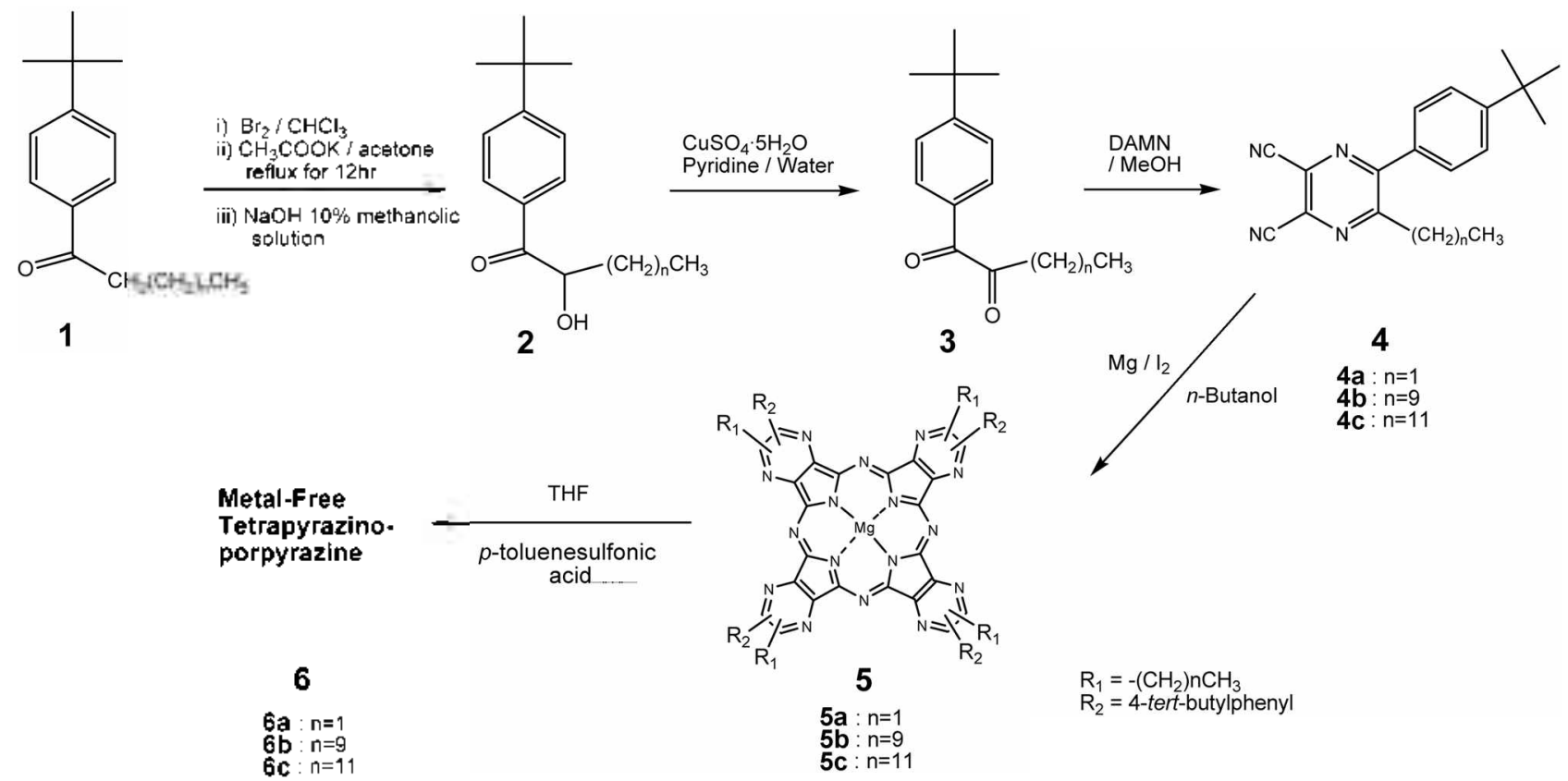

Scheme 1. Synthesis of tetrapyrazinoporphyrazine derivatives. 


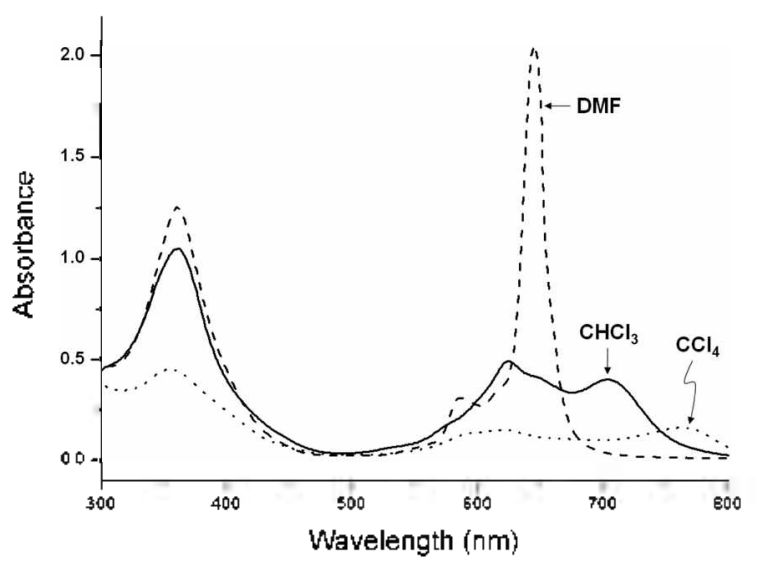

Figure 1. The effects of solvent polarities on the absontion spectra of $5 \mathbf{a}\left(2.52 \times 10^{-5} \mathrm{M}\right)$.

\section{in Scheme 1 .}

The absorption maxima in the electronic spectra of 5 and 6 in chloroform appeared at $625-705 \mathrm{~lm}$, due to a $\pi \cdot \pi^{\circ}$ transition, commonly referred to as the Q-band. The small broadness around $Q$ band region of $\mathbf{5}$ was generally observed because of structural mixtures resulting from their unsymmetrical structure. But the strange absorption curve observed in chlorinated solvent should be attributed to the molecular aggregation in solution (Figure 1).

Tetrapy razinoporplyrazinato magnesium complexes (5) in $N, N$-dimethylformanide (DMF) showed red fluorescence with small Stokes shift values. The fluorescence of $\mathbf{5 a}$ was greatly influenced by molecular aggregation. The Q-band spectrum causing the first $\pi \cdot \pi$ transition of $\mathbf{5}$ in DMF showed a pattern characteristic of a monomeric species, with a fluorescence maxima observed at $653.658 \mathrm{~nm}$, as shown in Figure 1. On the other hand, 5 a in chloroform did not show any fluorescence and had patterns characteristic of an aggregate, as shown in Figure 2.

The Q-band spectra of 6 in chlorofom and carbon tetrachloride showed patterns characteristic for a monomeric species with strong red fluorescence. However the absorption spectra of 6 in DMF did not show split $Q / Q$ bands. This is due to the electronic coupling between a pair (or

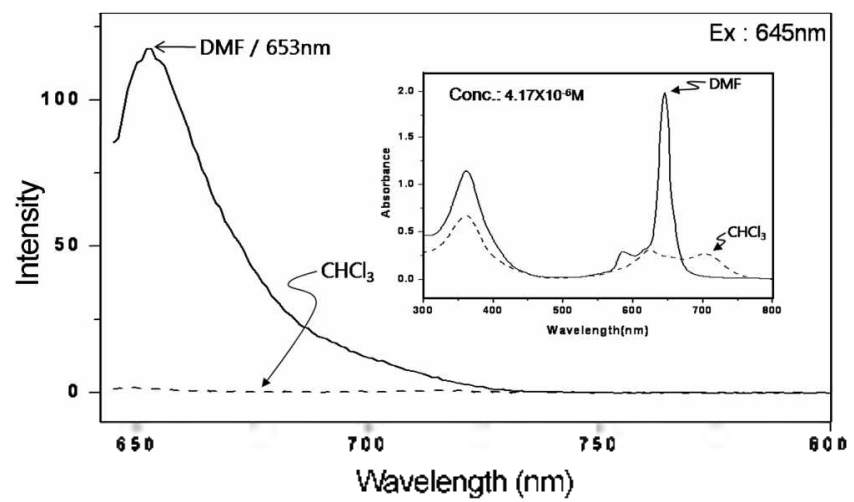

Figure 2. Emission spectra of $5 \mathbf{a}\left(4.17 \times 10^{-6} \mathrm{M}\right)$ in DMF and $\mathrm{CHCl}_{3}$. more) of porphyrazine units. ${ }^{11}$

In summary. we successfully synthesized organic-soluble tetrapyrazinoporphyrazines bearing long alkyl and $1 e r$-butyl phenyl substituents at peripheral positions. Porphyrazines 5 and 6 have satisfactory solubility in chlorinated hydrocarbons. THF. toluene. and $n$-hexane. but are practically insoluble in alcohols. Molecular aggregations and their functionalities will be reported elsewhere.

Acknowledgments. This work was supported by the research fund of Hanyang University (HY-2007-I).

\section{References}

1. Baik. B. Y.: Kwag. G.: Kim. S. Bull Korem Chent Soc. 2006. $27(2) .329$.

2. Rajagopal. G.: Kim. S. S.: Kwak. T. M. Bull. Korean Chent. Soc. 2006. 27(11). 1907

3. Templeton, D. H.; Fisher, M. S.; Zalkin, A.: Calvin. M. $d$. Am Chem. Soc 1971, $93(11) .2622$

4. Pawlowski. G.: Hanack. M. Sinthesis 1980. 4. 287.

5. Wheeler. B. L.: Nagasubramanian. G.: Bard. A. I.: Schechtman. L. A.: Kenney. M. E. J. Am. Chem. Soc. 1984. 106(24). 7404.

6. (a) Lee. B. H.; Jaung. J. Y. Dyes and Pigments 2003, $59(2), 135$. (b) Jaung. J. Y. Bull. Kor: Chem. Soc. 2004, 25(10), 1453. (c) Jaung. J. Y. Dyes and Pignents 2007, 75(2), 420.

7. Allegretti. M:: Bertini. R.: Cesta. M. C.: BiıŁarri. C.: Di Bitondo. R.: Di Cioceio. V: Galliera. E.: Berdini. V.: Topai. A.: Zampella. G.: Russo. V:: Di Bello. N.: Nano. G.: Nicolini. L.: Locati. M.: Fantucei, P.: Florio. S.: Colotta. F. J. Aled. Chem 2005, $18(13)$. 4312 .

8. Typical procedure to symthesize $5 \mathrm{a}$ : A suspension of $\mathrm{Mg}$ turnings ( $2 \mathrm{~g} .0 .08 \mathrm{~mol}$ ). one small erystal of iodine. and $n$-butanol (150 $\mathrm{mL}$ ) was heated under reflus for $4 \mathrm{~h}$. The reaction minture was then cooled to room temperature. and dicyanopyrazine $+\mathrm{a}(5.9 \mathrm{~g}$. $0.02 \mathrm{~mol}$ ) was added (all at once). The reaction mixture was quickly reheated to reflux. After approximately $10 \mathrm{~min}$, the reaction misture had become a dark green. After $\mathbf{l ~ h}$, the misture was quenched by adding methanol $(200 \mathrm{~mL})$. and filtered. yielding the crude product as a dark green solid. The crude product was purified by columnt chromatography. using silica gel as the stationary phase. and chloroform methanol (30:1) as the eluent. 5a (dark green solid, 240): $\left.{ }^{1} \mathrm{H}-\mathrm{NMR}(300 \mathrm{MHz}, \mathrm{CDCl})_{3}\right)$ d) 1.27 (broad s, $\mathrm{CH}_{3}, 12 \mathrm{H}$ ). 1.57 (broad s. $\left.\mathrm{C}\left(\mathrm{CH}_{3}\right)_{3}, 36 \mathrm{H}\right) .3 .66$ (broad s. $\mathrm{CH}_{2} .8 \mathrm{H}$ ). 7.79 (broad s. Ar-H. 8H). 8.10 (broad s. Ar-H. $8 \mathrm{H})$ : Anlal. caled. For $\mathrm{C}_{72} \mathrm{H}_{2} \mathrm{MgN}_{1}:$ C. $72.93:$ H. 6.12: N. 18.90 . Found: C. 73,07: H. 6.05: N. 18.74: MALDI-TOF mass-spectra: mz 1186.96 (cald. 1185.75).

9. Typical procedwe to symthesize ba: $p$-Toluenesulfonic acid $(27.5$ g. $0.16 \mathrm{~mol}$ ) was added to a solution of the $\mathrm{Mg}$ tetrapyrazinoporphyrazine (5a) $(3.7$ g. $3.1 \mathrm{~mm}$ ol) in tetrahydrofurat (THF. 100 $\mathrm{mL}$ ). and the resulting misture was stirred at room temperature for $30 \mathrm{~min}$. The solvent was removed in vacuo. yielding the crude product as a dark green solid. The crude product was purified by column chromatography. using silica gel as the stationary phase. and chloroformmethanol $(30: 1)$ as the eluent. 6a (dark green solid. 50\%): 'H-NMR (300 MHz. CDCl 3 ) $\delta:-1.47$ (s. N-H. $2 \mathrm{H}$ ). $1.51-1.54\left(\mathrm{ml} . \mathrm{C}\left(\mathrm{CH}_{3}\right) .36 \mathrm{H}\right) .1 .69-1.75\left(\mathrm{~m}_{\mathrm{CH}} \mathrm{CH}_{3} \mathrm{I} \mathrm{I} \mathrm{H}\right) .1 .76-1.79$ (mil. $\mathrm{CH}_{2} .8 \mathrm{H}$ ). 7.74 (broad s. Ar-H. $J=9.0 \mathrm{~Hz} .8 \mathrm{H}$ ). 8.08 (m. ArH. $J=9.0 \mathrm{~Hz}, 8 \mathrm{H}$ ): Anal. calcd. For $\mathrm{C}_{2} \mathrm{H}_{7} \mathrm{~N}_{16}: \mathrm{C} .74 .33: \mathrm{H}_{3} 6.41$; N. 19.26. Found: C, 74.63: H. 6.32: N. 19.08: MALDI-TOF massspectra: mz 1164.84 (calcd. 1163.47).

10. Mizuguchi. J.: Rihs. G.: Karfunkel. H. R. J. Phus Chent 1995. $99(44) .16217$. 